\title{
Navigating NSAID Use in Patients Receiving Oral Anticoagulation: Is There a Safe Course?
}

\author{
Anthony P. Kent ${ }^{1}$ \\ ${ }^{1}$ Department of Internal Medicine, Bridgeport Hospital, Yale New \\ Haven Health, Bridgeport, Connecticut, United States \\ Thromb Haemost 2020;120:1001-1003.
}

Address for correspondence Anthony P. Kent, MD, Department of Internal Medicine, Bridgeport Hospital, Yale New Haven Health, 1177 Post Road East APT 408 Westport, CT 06880, United States (e-mail: anthony.p.kent@gmail.com).
Oral anticoagulation (OAC) is used for thromboprophylaxis, but the downside is the risk of bleeding; hence, clinical decision-making often has to balance the reduction in thromboembolism against the potential for serious bleeding. ${ }^{1}$ Indeed, many clinical risk factors have been described as contributing to bleeding risk among OAC users. ${ }^{2}$ The risks of antithrombotic therapy-related bleeding are multifactorial, and even ethnicity may play a part. ${ }^{3}$

The use of aspirin or nonsteroidal anti-inflammatory drugs (NSAIDs) in combination with OAC poses a risk of bleeding. ${ }^{4-9}$ NSAIDs are also associated with thrombosis. ${ }^{6,8,10-14}$ From a clinical perspective, the chronic use of NSAIDs with OAC tends to intersect in patients with chronic osteoarthritis (OA) and atrial fibrillation (AF). The NSAID class has therapeutic benefit for treating arthritic pain and inflammation, and providing patients with important symptomatic relief and physical mobility. However, patients can develop serious major bleeding, in particular gastrointestinal (GI) major bleeding, when NSAIDs are combined with OAC.

Antiplatelet therapy (APT) or NSAID use in combination with warfarin are associated with an increased risk of major bleeding and GI major bleeding. ${ }^{4,6}$ The factor Xa inhibitors (apixaban, rivaroxaban) and direct thrombin inhibitor (dabigatran) have a safer overall intracranial bleeding profile relative to therapeutically dosed warfarin (international normalized ratio 2.03.0). ${ }^{15-17}$ Apixaban has been shown to have less major bleeding and GI major bleeding relative to warfarin. ${ }^{17}$ Dabigatran and rivaroxaban are associated with increased GI major bleeding, and relatively similar rates of major bleeding, relative to warfarin. ${ }^{15,16}$ NSAID use in combination with apixaban is associated with an increased risk of major bleeding, but not GI major bleeding. ${ }^{9}$ NSAID use in combination with dabigatran is associated with an increased risk of major bleeding and GI major bleeding. ${ }^{8}$ Rivaroxaban with NSAIDs has shown a similar trend. ${ }^{7}$ The general trends for major bleeding and GI major bleeding in combined NSAID and OAC use are summarized in - Table 1. In general, the use of NSAIDs in combination with the most commonly used OACs (apixaban, rivaroxaban, dabigatran, or warfarin) is limited by the risk of major bleeding.

The clinical question remains: is there a safe course for using NSAIDs in combination with OAC? Patients with AF on OAC and comorbid severe $\mathrm{OA}$ are limited in terms of pharmacologic $\mathrm{OA}$ relief, which can impair physical mobility and quality of life. Celecoxib, a selective COX-2 inhibitor NSAID, is effective for the symptomatic treatment of $\mathrm{OA}$ and improving physical function, ${ }^{18-20}$ and can be dosed once daily compared with multiple dosing for nonselective NSAIDs. Celecoxib is understudied in regards to safety when used with OAC (-Table $\mathbf{1}$ ).

In this issue of Thrombosis and Haemostasis, a meta-analysis by Zapata et al suggests that selective COX-2 inhibitors do not have an elevated risk of major bleeding, but do for GI major bleeding, when used in combination with warfarin, whereas nonselective NSAIDs were associated with an increased risk of both major bleeding and GI major bleeding. ${ }^{21}$ This analysis is consistent with a previous study regarding NSAID use with warfarin. ${ }^{6}$ In a previous large cohort analysis of NSAID use with warfarin, celecoxib was the only NSAID agent that did not demonstrate a significant increase in major bleeding when combined with OAC; however, selective COX-2 inhibitors as a class were associated with a significant increase in major bleeding and GI major bleeding. It is important to caution that nonselective NSAIDs and selective COX-2 inhibitors alone are associated with an increased bleeding and thrombotic risk compared with no NSAID use. ${ }^{6,12}$ Celecoxib was shown to have similar rates of major adverse cardiovascular events and GI bleeding risk compared with ibuprofen and naproxen in patients with $\mathrm{OA} .8,9,13,14$ Additional analysis of the selective COX-2 inhibitor celecoxib is needed to better understand its safety when used in combination with OAC.

Careful medication review is necessary to identify other bleeding risk agents, such as APT (e.g., aspirin, clopidogrel, ticagrelor), as well as multiple NSAID use, to avoid "stacking" bleeding risk. Review of the past medical history to gauge bleeding risk also helps to identify contraindications to NSAID received

May 4, 2020

accepted

May 4, 2020 (c) 2020 Georg Thieme Verlag KG Stuttgart · New York
DOI https://doi.org/ 10.1055/s-0040-1713098. ISSN 0340-6245. 
Table 1 General trends in bleeding, thrombosis among OACs with NSAIDs, and NSAIDs alone

\begin{tabular}{|c|c|c|c|c|}
\hline & Major bleeding & GI major bleeding & Thrombosis & Reference \\
\hline \multicolumn{5}{|l|}{ OAC with NSAID compared with no NSAID } \\
\hline Apixaban + Any NSAID & $\Uparrow$ & $\Leftrightarrow$ & $\Leftrightarrow$ & 12 \\
\hline Rivaroxaban + Any NSAID & $\Uparrow$ & $\Uparrow$ & - & 2 \\
\hline Dabigatran + Any NSAID & $\Uparrow$ & $\Uparrow$ & $\Uparrow$ & 3 \\
\hline Warfarin + Any NSAID & $\Uparrow$ & $\Uparrow$ & $\Uparrow$ & 1 \\
\hline Warfarin + Selective COX-2 inhibitors & $\Leftrightarrow$ & $\Uparrow$ & - & 1,16 \\
\hline Warfarin + Celecoxib & $\Leftrightarrow$ & - & - & 1 \\
\hline \multicolumn{5}{|c|}{ No OAC, NSAID alone compared with no NSAID } \\
\hline Any NSAID & $\Uparrow$ & $\Uparrow$ & $\Uparrow$ & 1,7 \\
\hline Selective COX-2 inhibitors & $\Uparrow$ & $\Uparrow$ & $\Uparrow$ & 1,7 \\
\hline Celecoxib & $\Uparrow$ & $\Uparrow$ & $\Uparrow$ & 1,7 \\
\hline
\end{tabular}

Abbreviations: COX-2, cyclooxygenase enzyme 2; GI, gastrointestinal; NSAID, nonsteroidal anti-inflammatory drug; OAC, oral anticoagulation. Note: $\Uparrow$, significant increase. $\Leftrightarrow$, no significant difference.

Table 2 Proposal for clinical assessment of NSAIDs in patients receiving OAC

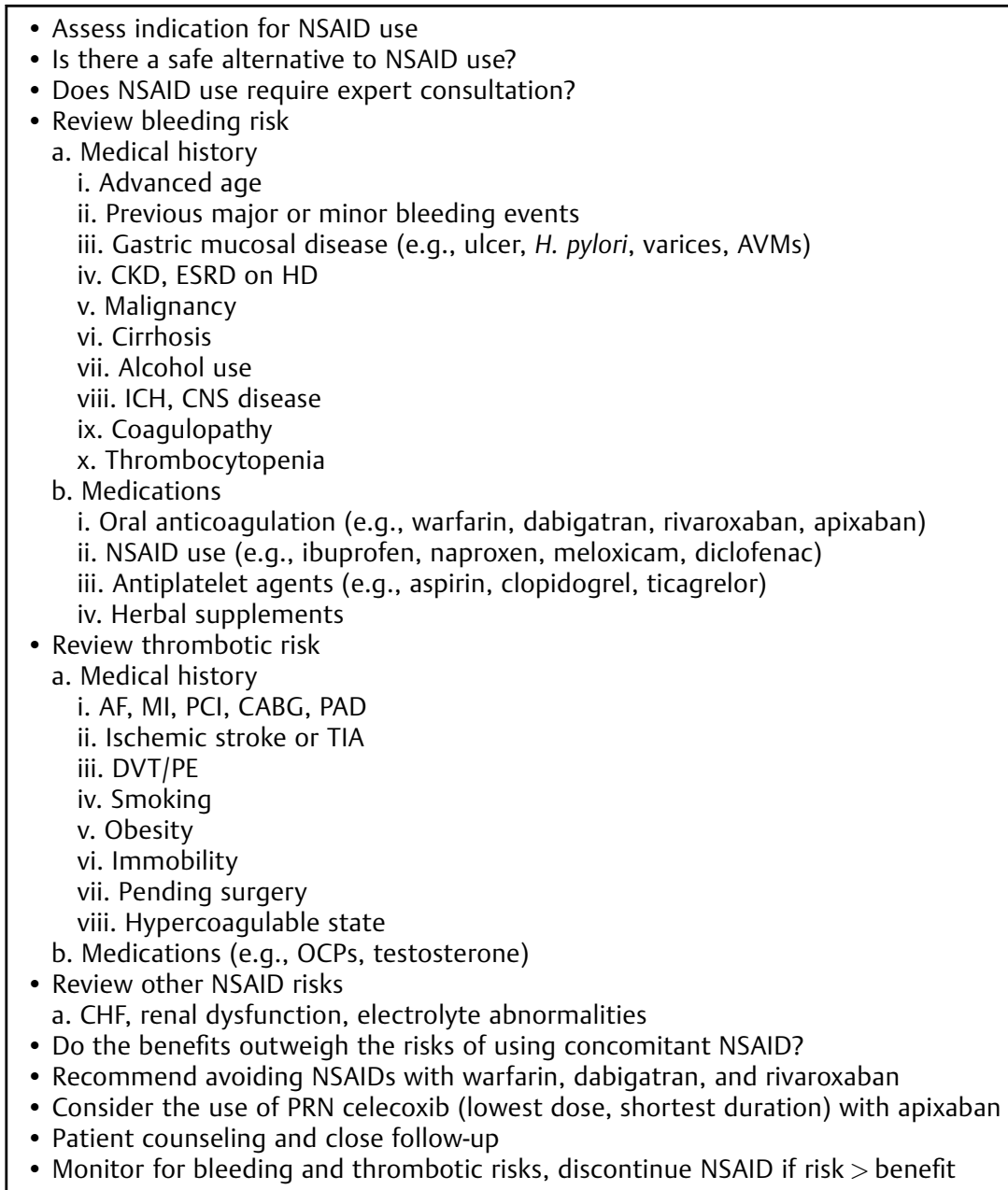

Abbreviations: AF, atrial fibrillation; AVM, arteriovenous malformation; CABG, coronary artery bypass graft; CHF, congestive heart failure; CKD, chronic kidney disease; CNS, central nervous system; DVT/PE, deep vein thrombosis/pulmonary embolism; ESRD, end-stage renal disease; HD, hemodialysis; ICH, intracranial hemorrhage; MI, myocardial infarction; NSAID, nonsteroidal anti-inflammatory drug; OAC, oral anticoagulation; OCPs, oral contraceptives; PAD, peripheral arterial disease; PCI, percutaneous coronary intervention; PRN, pro re nata; TIA, transient ischemic attack. 
use with OAC. Past medical history of major bleeding, GI mucosal disease, cirrhosis, or renal failure are major precautions to NSAID use all together. A proactive management strategy with appropriate use of dynamic bleeding risk assessment, mitigation of modifiable bleeding risk factors, and follow-up of high-risk patients was associated with lowered bleeding events and improved anticoagulation uptake among patients with AF, when compared with usual care. ${ }^{22}$

If patients with comorbid $\mathrm{AF}$ and $\mathrm{OA}$, receiving $\mathrm{OAC}$, are relatively otherwise healthy without bleed risk "stacking," then close clinical follow-up and patient counseling may warrant safe NSAID use on a pro re nata basis. A clinical approach to NSAIDs and OAC is proposed in - Table 2. Adjunctive analgesic therapies for $\mathrm{OA}$ include weight loss, physical therapy, acetaminophen, transdermal lidocaine, transdermal NSAIDs, and tramadol. Opioids are generally not recommended. Consultation with orthopaedics surgery for definitive arthroplasty is warranted in severe cases of OA. Duloxetine is recommended as a safe adjunctive therapy for chronic lumbar back pain. In summary, a comprehensive multimodal strategy for treating OA pain in patients with AF on OAC is recommended. Review with cardiology, rheumatology, hematology, or nephrology are also important considerations on a case-bycase basis before initiating an NSAID in patients receiving OAC.

Importantly, NSAIDs are also used on a short-term basis for their antipyretic effects. It is important to opt for acetaminophen in place of NSAIDs for patients with a febrile illness who are on OAC. NSAID-related renal dysfunction is also an important consideration, especially in regards to the pharmacokinetics of OACs that depend on the creatinine clearance (renal elimination: apixaban $27 \%$, rivaroxaban $66 \%$, dabigatran $80 \%$ ).

Further clinical investigation into the relationship between NSAID and OAC use, specifically the selective COX-2 inhibitor celecoxib at low dose, may help define a relatively safe strategy for their combined use in select situations to address the therapeutic goals of patients with comorbid $\mathrm{OA}$ and $\mathrm{AF}$, among other related chronic conditions in which NSAIDs and OAC intersect.

\section{Conflict of Interest}

None declared.

\section{References}

1 Proietti M, Mujovic N, Potpara TS. Optimizing stroke and bleeding risk assessment in patients with atrial fibrillation: a balance of evidence, practicality and precision. Thromb Haemost 2018;118 (12):2014-2017

2 Kim K, Yamashita Y, Morimoto T, et al; COMMAND VTE Registry Investigators. Risk factors for major bleeding during prolonged anticoagulation therapy in patients with venous thromboembolism: from the COMMAND VTE Registry. Thromb Haemost 2019; 119(09):1498-1507

3 Kang J, Park KW, Palmerini T, et al. Racial differences in ischaemia/bleeding risk trade-off during anti-platelet therapy: individual patient level landmark meta-analysis from seven RCTs. Thromb Haemost 2019;119(01):149-162

4 Lamberts M, Gislason GH, Lip GY, et al. Antiplatelet therapy for stable coronary artery disease in atrial fibrillation patients taking an oral anticoagulant: a nationwide cohort study. Circulation 2014;129(15):1577-1585

5 Heidbuchel H, Verhamme P, Alings M, et al; ESC Scientific Document Group. Updated European Heart Rhythm Association practical guide on the use of non-vitamin-K antagonist anticoagulants in patients with non-valvular atrial fibrillation: executive summary. Eur Heart J 2017;38(27):2137-2149

6 Lamberts M, Lip GY, Hansen ML, et al. Relation of nonsteroidal anti-inflammatory drugs to serious bleeding and thromboembolism risk in patients with atrial fibrillation receiving antithrombotic therapy: a nationwide cohort study. Ann Intern Med 2014; 161(10):690-698

7 Davidson BL, Verheijen S, Lensing AW, et al. Bleeding risk of patients with acute venous thromboembolism taking nonsteroidal anti-inflammatory drugs or aspirin. JAMA Intern Med 2014; 174(06):947-953

8 Kent AP, Brueckmann M, Fraessdorf M, et al. Concomitant oral anticoagulant and nonsteroidal anti-inflammatory drug therapy in patients with atrial fibrillation.J Am Coll Cardiol 2018;72(03):255-267

9 Dalgaard F, Mulder H, Wojdyla DM, et al. Patients with atrial fibrillation taking nonsteroidal anti-inflammatory drugs and oral anticoagulants in the ARISTOTLE trial. Circulation 2020;141(01): $10-20$

10 Schmidt M, Christiansen CF, Horváth-Puhó E, Glynn RJ, Rothman KJ, Sørensen HT. Non-steroidal anti-inflammatory drug use and risk of venous thromboembolism. J Thromb Haemost 2011;9(07): 1326-1333

11 Salvo F, Pariente A, Noize P, Fourrier-Réglat A, Moore N. NSAIDs discontinuation and myocardial infarction. J Thromb Haemost 2009;7(09):1600-1601

12 Coxib and traditional NSAID Trialists' (CNT) Collaboration, Bhala $\mathrm{N}$, Emberson J, Merhi A, et al. Vascular and upper gastrointestinal effects of non-steroidal anti-inflammatory drugs: meta-analyses of individual participant data from randomised trials. Lancet 2013;382(9894):769-779

13 Nissen SE, Yeomans ND, Solomon DH, et al; PRECISION Trial Investigators. Cardiovascular safety of celecoxib, naproxen, or ibuprofen for arthritis. N Engl J Med 2016;375(26):2519-2529

14 Solomon DH, Husni ME, Libby PA, et al. The risk of major NSAID toxicity with celecoxib, ibuprofen, or naproxen: a secondary analysis of the PRECISION trial. Am J Med 2017;130(12):1415-1422

15 Connolly SJ, Ezekowitz MD, Yusuf S, et al; RE-LY Steering Committee and Investigators. Dabigatran versus warfarin in patients with atrial fibrillation. N Engl J Med 2009;361(12):1139-1151

16 Patel MR, Mahaffey KW, Garg J, et al; ROCKET AF Investigators. Rivaroxaban versus warfarin in nonvalvular atrial fibrillation. $\mathrm{N}$ Engl J Med 2011;365(10):883-891

17 Granger CB, Alexander JH, McMurray JJ, et al; ARISTOTLE Committees and Investigators. Apixaban versus warfarin in patients with atrial fibrillation. N Engl J Med 2011;365(11):981-992

$18 \mathrm{Xu} \mathrm{C}, \mathrm{Gu}$ K, Yasen Y, Hou Y. Efficacy and safety of celecoxib therapy in osteoarthritis: a meta-analysis of randomized controlled trials. Medicine (Baltimore) 2016;95(20):e3585

19 Gordo AC, Walker C, Armada B, Zhou D. Efficacy of celecoxib versus ibuprofen for the treatment of patients with osteoarthritis of the knee: a randomized double-blind, non-inferiority trial. J Int Med Res 2017;45(01):59-74

20 Puljak L, Marin A, Vrdoljak D, Markotic F, Utrobicic A, Tugwell P. Celecoxib for osteoarthritis. Cochrane Database Syst Rev 2017;5: CD009865

21 Zapata L, Hansten P, Panic J, et al. Risk of bleeding with exposure to warfarin and non-steroidal anti-inflammatory drugs: a systematic review and meta-analysis. Thromb Haemost 2020;120(07):1066-1074

22 Guo Y, Lane DA, Chen Y, et al. Regular bleeding risk assessment associated with reduction in bleeding outcomes: the mAFA II randomised trial. Am J Med 2020. Doi: 10.1016/j.amjmed.2020.03.019 